\title{
Adsorption Equilibria of C1-C4 from natural gas on graphene sheets
}

\author{
Xueli Zhang ${ }^{a}$, Yan Sun ${ }^{a}$, Wei Su ${ }^{b}$ and Xueyuan Wang ${ }^{\mathrm{c}}$
}

${ }^{\mathrm{a} D e p a r t m e n t ~ o f ~ C h e m i s t r y, ~ S c h o o l ~ o f ~ S c i e n c e, ~ T i a n j i n ~ U n i v e r s i t y, ~ T i a n j i n ~ 300072, ~ P R ~}$

China

${ }^{\mathrm{b}}$ School of Chemical Engineering and Technology, Tianjin University, Tianjin

300072, PR China

${ }^{c}$ Huajin Pharmaceutical Co,. Ltd, Tianjin 300241, PR China

The corresponding author email address: sunyan2011@tju.edu.cn.

Table S1. Adsorption data of $\mathrm{CH}_{4}$ on GS

\begin{tabular}{|c|c|c|c|c|c|c|c|c|c|}
\hline \multicolumn{2}{|c|}{$263 \mathrm{~K}^{\mathrm{a}}$} & \multicolumn{2}{|r|}{$273 \mathrm{~K}^{\mathrm{a}}$} & \multicolumn{2}{|c|}{$283 \mathrm{~K}^{\mathrm{a}}$} & \multicolumn{2}{|c|}{$293 K^{a}$} & \multicolumn{2}{|r|}{$303 \mathrm{~K}^{\mathrm{a}}$} \\
\hline $\mathrm{p}^{\mathrm{b}}$ & $\mathrm{n}^{\mathrm{c}}$ & $\mathrm{p}^{\mathrm{b}}$ & $\mathrm{n}^{\mathrm{c}}$ & $p^{b}$ & $\mathrm{n}^{\mathrm{c}}$ & $\mathrm{p}^{\mathrm{b}}$ & $\mathrm{n}^{\mathrm{c}}$ & $\mathrm{p}^{\mathrm{b}}$ & $\mathrm{n}^{\mathrm{c}}$ \\
\hline Mpa & $\mathrm{mmol} / \mathrm{g}$ & Mpa & $\mathrm{mmol} / \mathrm{g}$ & Mpa & $\mathrm{mmol} / \mathrm{g}$ & Mpa & $\mathrm{mmol} / \mathrm{g}$ & Mpa & $\mathrm{mmol} / \mathrm{g}$ \\
\hline 0.22537 & 0.93602 & 0.19924 & 0.6652 & 0.23657 & 0.72391 & 0.24404 & 0.52969 & 0.22164 & 0.41705 \\
\hline 0.54832 & 1.65595 & 0.75926 & 1.74106 & 0.60992 & 1.32046 & 0.56139 & 1.03026 & 0.65473 & 0.95332 \\
\hline 0.99074 & 2.24564 & 1.41263 & 2.42231 & 1.10275 & 1.85684 & 0.92727 & 1.42475 & 1.01688 & 1.25341 \\
\hline 1.54832 & 2.78469 & 2.03239 & 2.84031 & 1.74118 & 2.2987 & 1.5545 & 1.85545 & 1.62171 & 1.67809 \\
\hline 2.24147 & 3.17717 & 2.73803 & 3.19600 & 2.46922 & 2.7271 & 2.0604 & 2.19139 & 2.28254 & 1.98229 \\
\hline 2.98818 & 3.49017 & 3.51087 & 3.47797 & 3.25699 & 2.97387 & 2.70443 & 2.52253 & 2.97324 & 2.2557 \\
\hline 3.72741 & 3.76031 & 4.29677 & 3.60140 & 4.15677 & 3.17114 & 3.34286 & 2.8185 & 3.74421 & 2.44526 \\
\hline 4.55252 & 3.94027 & 4.78026 & 3.60192 & 4.98374 & 3.29505 & 4.26131 & 2.95348 & 4.42371 & 2.59115 \\
\hline 5.17228 & 4.02756 & 5.90405 & 3.68219 & 5.63711 & 3.32493 & 5.27682 & 3.07344 & 5.16108 & 2.68764 \\
\hline 5.68004 & 4.0667 & & & & & & & 5.72858 & 2.69805 \\
\hline
\end{tabular}

Table S2. Adsorption data of $\mathrm{C}_{2} \mathrm{H}_{6}$ on GS 


\begin{tabular}{|c|c|c|c|c|c|c|c|c|c|}
\hline \multicolumn{2}{|c|}{$263 \mathrm{~K}^{\mathrm{a}}$} & \multicolumn{2}{|r|}{$273 K^{a}$} & \multicolumn{2}{|c|}{$283 \mathrm{~K}^{\mathrm{a}}$} & \multicolumn{2}{|c|}{$293 K^{a}$} & \multicolumn{2}{|r|}{$303 \mathrm{~K}^{\mathrm{a}}$} \\
\hline $\mathrm{p}^{\mathrm{b}}$ & $\mathrm{n}^{\mathrm{c}}$ & $p^{b}$ & $\mathrm{n}^{\mathrm{c}}$ & $p^{b}$ & $\mathrm{n}^{\mathrm{c}}$ & $\mathrm{p}^{\mathrm{b}}$ & $\mathrm{n}^{\mathrm{c}}$ & $\mathrm{p}^{\mathrm{b}}$ & $\mathrm{n}^{\mathrm{c}}$ \\
\hline Mpa & $\mathrm{mmol} / \mathrm{g}$ & Mpa & $\mathrm{mmol} / \mathrm{g}$ & Mpa & $\mathrm{mmol} / \mathrm{g}$ & Mpa & $\mathrm{mmol} / \mathrm{g}$ & Mpa & $\mathrm{mmol} / \mathrm{g}$ \\
\hline 0.04597 & 1.16822 & 0.04779 & 1.31275 & 0.04835 & 1.12523 & 0.04767 & 0.76885 & 0.04892 & 0.77455 \\
\hline 0.25897 & 2.96778 & 0.25897 & 2.79986 & 0.23657 & 2.31026 & 0.30378 & 2.43076 & 0.30378 & 2.13248 \\
\hline 0.49418 & 3.76401 & 0.47925 & 3.4418 & 0.43071 & 2.93251 & 0.56512 & 3.04067 & 0.56139 & 2.73066 \\
\hline 0.7462 & 4.45597 & 0.66779 & 3.84892 & 0.63419 & 3.3272 & 0.81153 & 3.42922 & 0.81901 & 3.11725 \\
\hline 0.96461 & 5.03558 & 0.85074 & 4.26559 & 0.82647 & 3.68312 & 1.03368 & 3.72476 & 1.02994 & 3.38461 \\
\hline 1.16062 & 5.64736 & 1.03555 & 4.63744 & 1.04488 & 4.03749 & 1.21475 & 3.94041 & 1.23529 & 3.58734 \\
\hline 1.34543 & 6.32789 & 1.25209 & 5.1192 & 1.22595 & 4.37605 & 1.38836 & 4.16606 & 1.42383 & 3.79898 \\
\hline 1.51717 & 6.99786 & 1.43503 & 5.52313 & 1.42756 & 4.73841 & 1.57317 & 4.40872 & 1.61424 & 3.93912 \\
\hline & & 1.57691 & 5.84862 & 1.59557 & 4.96982 & & & & \\
\hline
\end{tabular}

${ }^{\mathrm{a}}$ Measured with $0.1 \mathrm{~K}$ uncertainty. ${ }^{\mathrm{b}}$ Measured with $0.6 \mathrm{kPa}$ uncertainty. ${ }^{\mathrm{c} C a l c u l a t e d}$ with $9.3 \cdot 10^{-3} \mathrm{mmol} / \mathrm{g}$ uncertainty.

\section{Table S3. Adsorption data of $\mathrm{C}_{3} \mathrm{H}_{8}$ on GS}

\begin{tabular}{|c|c|c|c|c|c|c|c|c|c|}
\hline \multicolumn{2}{|c|}{$263 \mathrm{~K}^{\mathrm{a}}$} & \multicolumn{2}{|r|}{$273 \mathrm{~K}^{\mathrm{a}}$} & \multicolumn{2}{|c|}{$283 \mathrm{~K}^{\mathrm{a}}$} & \multicolumn{2}{|c|}{$293 K^{a}$} & \multicolumn{2}{|c|}{$303 \mathrm{~K}^{\mathrm{a}}$} \\
\hline $\mathrm{p}^{\mathrm{b}}$ & $\mathrm{n}^{\mathrm{c}}$ & $\mathrm{p}^{\mathrm{b}}$ & $\mathrm{n}^{\mathrm{c}}$ & $\mathrm{p}^{\mathrm{b}}$ & $\mathrm{n}^{\mathrm{c}}$ & $\mathrm{p}^{\mathrm{b}}$ & $\mathrm{n}^{\mathrm{c}}$ & $\mathrm{p}^{\mathrm{b}}$ & $\mathrm{n}^{\mathrm{c}}$ \\
\hline Mpa & $\mathrm{mmol} / \mathrm{g}$ & Mpa & $\mathrm{mmol} / \mathrm{g}$ & Mpa & $\mathrm{mmol} / \mathrm{g}$ & Mpa & $\mathrm{mmol} / \mathrm{g}$ & Mpa & $\mathrm{mmol} / \mathrm{g}$ \\
\hline 0.00678 & 0.31577 & 0.01043 & 0.32182 & 0.01356 & 0.28377 & 0.01356 & 0.27377 & 0.01564 & 0.14391 \\
\hline 0.02033 & 0.91259 & 0.02711 & 0.89209 & 0.03233 & 0.87825 & 0.03233 & 0.77825 & 0.03285 & 0.66736 \\
\hline 0.05423 & 1.58045 & 0.06153 & 1.47124 & 0.06987 & 1.41704 & 0.06987 & 1.31704 & 0.07247 & 1.23597 \\
\hline 0.0975 & 2.24914 & 0.10845 & 1.99945 & 0.12044 & 1.96165 & 0.12044 & 1.76165 & 0.12514 & 1.55996 \\
\hline 0.14286 & 3.1368 & 0.15642 & 2.51678 & 0.17363 & 2.40351 & 0.17363 & 2.20351 & 0.18249 & 1.99262 \\
\hline 0.18562 & 3.96116 & 0.20595 & 3.0799 & 0.22577 & 2.88646 & 0.22577 & 2.58646 & 0.24349 & 2.27528 \\
\hline 0.22889 & 4.82235 & 0.25653 & 3.66808 & 0.27947 & 3.40166 & 0.27947 & 3.00166 & 0.2972 & 2.5106 \\
\hline 0.26696 & 5.65206 & 0.29355 & 4.26082 & & & & & & \\
\hline 0.29407 & 6.32334 & & & & & & & & \\
\hline
\end{tabular}

${ }^{\mathrm{a}}$ Measured with $0.1 \mathrm{~K}$ uncertainty. ${ }^{\mathrm{b}}$ Measured with $0.6 \mathrm{kPa}$ uncertainty. ${ }^{\mathrm{c}}$ Calculated with $9.3 \cdot 10^{-3} \mathrm{mmol} / \mathrm{g}$ uncertainty.

Table S4. Adsorption data of $\mathrm{C}_{4} \mathrm{H}_{10}$ on GS

\begin{tabular}{|c|c|c|c|c|c|c|c|c|c|}
\hline \multicolumn{2}{|r|}{$263 \mathrm{~K}^{\mathrm{a}}$} & \multicolumn{2}{|r|}{$273 \mathrm{~K}^{\mathrm{a}}$} & \multicolumn{2}{|c|}{$283 \mathrm{~K}^{\mathrm{a}}$} & \multicolumn{2}{|c|}{$293 \mathrm{~K}^{\mathrm{a}}$} & \multicolumn{2}{|r|}{$303 \mathrm{~K}^{\mathrm{a}}$} \\
\hline $\mathrm{p}^{\mathrm{b}}$ & $\mathrm{n}^{\mathrm{c}}$ & $\mathrm{p}^{\mathrm{b}}$ & $\mathrm{n}^{\mathrm{c}}$ & $\mathrm{p}^{\mathrm{b}}$ & $\mathrm{n}^{\mathrm{c}}$ & $\mathrm{p}^{\mathrm{b}}$ & $\mathrm{n}^{\mathrm{c}}$ & $\mathrm{p}^{\mathrm{b}}$ & $\mathrm{n}^{\mathrm{c}}$ \\
\hline Мpa & $\mathrm{mmol} / \mathrm{g}$ & Mpa & $\mathrm{mmol} / \mathrm{g}$ & Mpa & $\mathrm{mmol} / \mathrm{g}$ & Mpa & $\mathrm{mmol} / \mathrm{g}$ & Mpa & $\mathrm{mmol} / \mathrm{g}$ \\
\hline 0.00052 & 0.15437 & 0.00261 & 0.21516 & 0.00678 & 0.23082 & 0.00209 & 0.05441 & 0.00469 & 0.03595 \\
\hline 0.00313 & 0.35390 & 0.00521 & 0.39153 & 0.01147 & 0.38593 & 0.00626 & 0.14786 & 0.00834 & 0.12083 \\
\hline 0.00678 & 0.62515 & 0.01043 & 0.6097 & 0.02033 & 0.73937 & 0.00991 & 0.29007 & 0.01199 & 0.25823 \\
\hline 0.01199 & 0.95209 & 0.01460 & 0.89087 & 0.03389 & 1.05001 & 0.01512 & 0.45224 & 0.01721 & 0.41924 \\
\hline 0.01773 & 1.34532 & 0.02294 & 1.15187 & 0.04849 & 1.28264 & 0.02242 & 0.65096 & 0.02398 & 0.58796 \\
\hline 0.02398 & 1.77665 & 0.03024 & 1.46073 & & & 0.03233 & 0.82930 & 0.03389 & 0.75556 \\
\hline 0.03076 & 2.24664 & 0.03806 & 1.79120 & & & 0.04171 & 0.98411 & 0.04953 & 0.85037 \\
\hline 0.03650 & 2.78731 & 0.04797 & 2.17167 & & & 0.05110 & 1.09011 & & \\
\hline
\end{tabular}

